\title{
Application of Improved Droop Control Based on Grid Impedance in Photovoltaic Grid-Connected Power Generation system
}

\author{
$\mathrm{Xu} \mathrm{HaoHao}{ }^{1, *}$, Wang Fang ${ }^{1}$, Liang ZhiCheng ${ }^{1}$ \\ ${ }^{1}$ Shanghai DianJi University, Shanghai 201306, China
}

\begin{abstract}
When the power grid impedance mutates, the output power of the grid-connected photovoltaic power generation system controlled by drooping will become very unstable. To solve this problem, an improved droop control algorithm based on network impedance detection is proposed. Taking photovoltaic grid-connected power generation system as the research object, the influence of power grid impedance on traditional droop control is firstly expounded by introducing power grid impedance value into traditional droop control. Then the method of real-time network impedance extraction is introduced. Furthermore, according to the bandwidth and phase angle constraints of the equivalent output impedance phase frequency characteristics of the photovoltaic grid-connected inverter, the controller parameters are updated in real time to achieve the purpose of adaptive network impedance mutation. Finally, the proposed method is tested and verified by real-time simulation in the loop respectively. The results show that the proposed method is correct.
\end{abstract}

\section{Introduction}

Power grid impedance is widely found in power systems. It is usually composed of leakage reactance of transformers and parasitic impedance of transmission lines. It is an equivalent impedance that exists naturally and cannot be ignored ${ }^{[1-3]}$. Many scholars have made indepth analysis on the influence of grid impedance on the control performance of grid-connected inverter. Literature [4] established the equivalent circuit model of the threephase LCL grid-connected inverter parallel system. According to the established equivalent circuit model, the influence of power grid impedance on the current control of grid-connected inverter in large photovoltaic power stations was analysed by using the traditional frequency domain analysis method. Literature [5] established the Norton equivalent model of the three-phase inverter parallel system and derived the expressions of gridconnected current and grid-connected common point voltage of the inverter. Finally, the root locus method was used to analyse the influence of grid impedance on the stability of large-scale grid-connected photovoltaic system. The analysis results of the above literatures all indicate that the existence of network impedance will have a certain influence on the output electric energy of the grid-connected inverter connected with it, and even cause serious distortion of grid-connected voltage and significant increase of harmonic content, thus affecting the quality of grid-connected electric energy. Especially for the weak grid, the grid impedance has a greater impact on the grid-connected voltage. However, the above literatures only analysed the influence of power grid impedance on the stability of grid-connected system, but did not propose corresponding solutions.

In order to explore the influence of power grid impedance on traditional droop control, the real-time power grid impedance value is firstly introduced into the traditional droop control, and the theoretical analysis is carried out. Then the measurement technology of power grid impedance is studied deeply, and the power grid impedance extraction method based on DFT is introduced. Then, based on the equivalent output impedance of the grid-connected photovoltaic inverter and the bandwidth and phase Angle constraints in the phase frequency characteristics, the real-time update of the control parameters in the PI controller was calculated, and the improved drooping control algorithm based on the grid impedance detection was realized. Finally, the improved droop control algorithm is tested and verified on RT-LAB semi-physical real-time simulator respectively.

\section{Influence of power network impedance on traditional droop control}

Droop control is based on the simulation of droop characteristics of synchronous generator P-F and Q-U, and according to the active and reactive power output by the current grid-connected inverter. The given voltage reference value is adjusted in real time, and then the voltage reference value is converted into the output voltage of the grid-connected inverter by the double-loop voltage and current controller. Finally, the module generated by SPWM is converted into control signal to the

\footnotetext{
* Corresponding author: Haohao Xu, E-mail: xhh47741@163.com
} 
IGBT switch tube, so as to realize the purpose of controlling the output voltage and power of the gridconnected inverter.

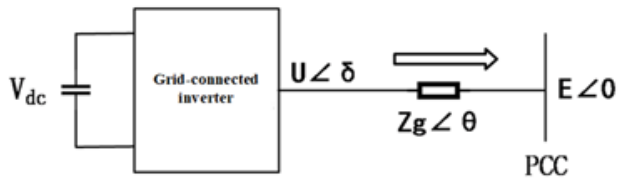

Figure1. Equivalent model of power transmission for gridconnected power generation system

In Figure1, $\mathrm{U} \angle \delta$ is the output voltage of the gridconnected inverter with amplitude $U$ and phase angle $\delta$; $\mathrm{Zg} \angle \theta$ is the equivalent network impedance with impedance value of $\mathrm{Zg}$ and impedance angle $\theta ; \mathrm{E} \angle 0$ is the grid side voltage with amplitude $E$ and phase Angle 0.

From Figure1, the output power expression of the gridconnected inverter as shown in formula (1) can be derived:

$$
S=P+j Q=E^{*}\left(\frac{U \cos \delta+j U \sin \delta-E}{Z \cos \theta+j Z \sin \theta}\right)^{*}
$$

According to Formula (1), the active power and reactive power output by the grid-connected inverter of Formula (2) can be obtained as follows:

$$
\begin{aligned}
& \text { 衰 } P=\left(\frac{E U}{Z} \cos \delta-\frac{E^{2}}{Z}\right) \cos \theta+\frac{E U}{Z} \sin \delta \sin \theta \\
& \text { 美 } Q=\left(\frac{E U}{Z} \cos \delta-\frac{E^{2}}{Z}\right) \sin \theta+\frac{E U}{Z} \sin \delta \cos \theta
\end{aligned}
$$

It is easy to find that if the impedance of the grid is $\mathrm{Lg}>>\mathrm{Rg}$, that is, in the case of strong grid, it is $\mathrm{Zg} \approx \mathrm{Xg}$, $\theta \approx 90^{\circ}$. Generally speaking, the phase Angle of output voltage of grid-connected inverter is very different from the phase Angle of grid voltage, so $\sin \delta \approx \delta, \cos \delta \approx 1$ when $\delta \approx 0^{\circ}$. Accordingly, equation (2) can be simplified to the following equation:

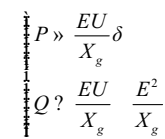

It can be seen from Equation (3) that it conforms to the P-f and Q-U characteristics in the droop control. When the grid impedance $\mathrm{Lg}<<\mathrm{Rg}$, namely the situation of weak grid, at this time, $\mathrm{Zg} \approx \operatorname{Rg}$ and $\theta \approx 0^{\circ}$. Set $\delta \approx 0^{\circ}$, thereby $\sin \delta \approx \delta, \quad \cos \delta \approx 1$. Accordingly, equation (3) can be simplified to the following equation:

$$
\begin{aligned}
& P ? \frac{E U}{R_{\mathrm{g}}} \frac{E^{2}}{R_{\mathrm{g}}} \\
& \frac{1}{1} Q » \frac{E U}{R_{\mathrm{g}}} \delta
\end{aligned}
$$

It can be seen from the equation (4), when the power grid impedance is no longer present perceptual and present resistance, drooping at this time no longer meet the traditional droop characteristic, namely USES the original droop control method at this time, will not be able to effectively control the grid voltage and the output of active power and reactive power, will ultimately lead to a serious fault and shut down the grid-connected power generation system.

\section{Real-time impedance detection method based on photovoltaic grid-connected inverter}

According to the above section, when the impedance of the power grid changes from sensibility to resistance, if the impedance value of the power grid can be detected in real time, it may be helpful to keep the impedance of the power grid as sensibility. So-called grid impedance detection based on photovoltaic grid inverter, which is to point to by photovoltaic grid inverter to the grid system with certain specific harmonic current, then through such as fast Fourier transform (FFT), the extracted after grid impedance of harmonic voltage current. Finally, the size of grid impedance in electric power system can be calculated with a certain formula.

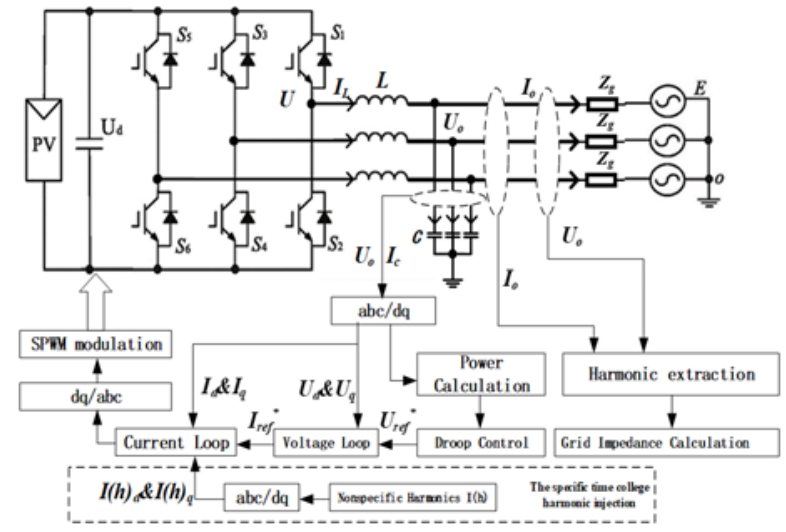

Figure2. Block diagram of grid impedance detection with photovoltaic grid-connected inverter

For disturbance injection, the most important thing is not to destroy the stability of the original system. Noncharacteristic subharmonic is a kind of power grid harmonic whose frequency is $0 \sim 1$ times of the fundamental wave frequency. Because there are a large number of odd harmonics in the power system, but the content of non-characteristic subharmonics is very small, it can be easily extracted from the power system by injecting a small amount of non-characteristic subharmonics without affecting the power quality of the power system. A non-characteristic subharmonic with a frequency of $75 \mathrm{HZ}$ is selected in this paper, and its amplitude is $10 \%$ of the grid-connected current. In addition, the method of disturbance injection is required to be simple and feasible.

The method with good effect and easy implementation is selected to add perturbations to a given current. Figure 2 shows the block diagram of the grid impedance detection structure based on the grid-connected photovoltaic inverter.

Generally, the method commonly used at present to quickly extract the harmonic voltage and current on the grid impedance is called Discrete Fourier Transform (DFT). Its discrete computation property can effectively speed up the operation speed of the controller of grid- 
connected inverter. For any periodic signal $\mathrm{x}(\mathrm{kT})$ (such as voltage, current, etc.), it can be expressed as the sum of harmonic signals of each order by DFT formula. Taking the $75 \mathrm{HZ}$ harmonic $\Lambda_{75 \mathrm{HZ}}$ in this paper as an example, the calculation formula shown in formula (5) can be obtained.

$$
\Lambda_{75 H Z}=\sum_{k=0}^{N-1} v(k) \cos \left(\frac{2 \pi \mathrm{h} k}{N}\right)+j \sum_{k=0}^{N-1} v(k) \sin \left(\frac{2 \pi \mathrm{h} k}{N}\right)
$$

where $\mathrm{v}(\mathrm{k})$-- is the input signal (such as voltage, current, etc.) at time $\mathrm{K}$;

$\mathrm{h}$-- is the number of certain harmonic vibrations within the sampling period; $h$ can be obtained by the product of sampling time and harmonic frequency.

$\mathrm{N}$-- is the number of samples, can be calculated by the ratio of sampling duration to sampling frequency;

$\mathrm{k}$-- represents the current number of sampling points, $\mathrm{k}=0,1, \ldots, \mathrm{N}-1$.

Finally, the amplitudes and phase angles of the voltage and current signals are further processed to obtain realtime network impedance values. The calculation formula is shown in Equation (6):

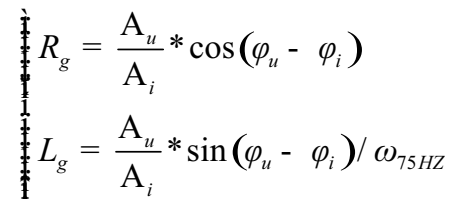

where $\mathrm{A}_{u}$ and $\mathrm{A}_{i}$-- are the amplitude of harmonic current and harmonic voltage respectively;

$\varphi_{u}$ and $\varphi_{i} \quad-$ are the phase angles of harmonic current and harmonic voltage respectively;

$\omega_{75 \mathrm{HZ}} \quad--$ is the angular frequency of $75 \mathrm{HZ}$ harmonic.

\section{Improved droop control principle based on network impedance detection}

In order to study the control model of drooping control after the introduction of network impedance, the equations as shown in equation (7) are first obtained according to Figure 2 and the voltage-current relationship of the filter capacitance and inductance, as well as the network impedance.

$$
\begin{aligned}
& U_{0}=U_{c f}-U_{z} \\
& U_{\mathrm{dc}}=U_{L f}+U_{C f} \\
& \frac{1}{U_{z}}=L_{g} \cdot \frac{d I_{0}}{d t}+R_{g} I_{o} \\
& I_{L f}=I_{C f}+I_{0}
\end{aligned}
$$

where Ucf and Ucf, Icf and Icf - are the voltage and current on capacitance and inductance;

Udc -- is DC bus voltage;

$\mathrm{Uz}$-- is the output voltage on the network impedance;

U0 and I0 -- are output voltage and current of photovoltaic grid-connected inverter after passing LC filter;
According to Equation (7), the structure diagram of voltage and current double-loop control system with improved droop control is designed, as shown in Figure3.

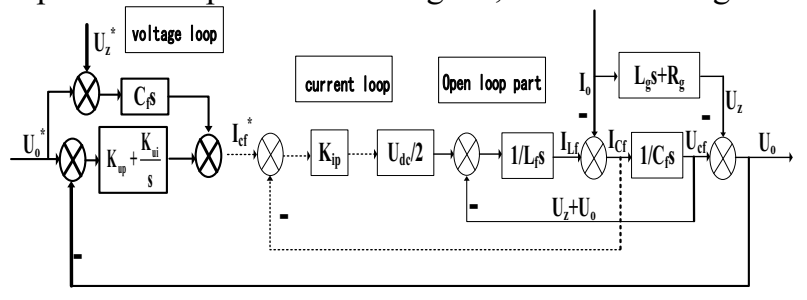

Figure 3. Structure diagram of voltage and current double loop control system for improved droop control

In Figure3, the thickened solid line part is the voltage loop control structure, the dashed line part is the current loop control structure, and the rest part is the system openloop control structure. Where, Icf* represents the reference value of capacitance current obtained through the voltage loop calculation; $\mathrm{I} / \mathrm{O}$ as disturbance input; Uo* and $\mathrm{Uz}^{*}$ represent grid reference voltage and grid impedance voltage respectively. Where, after the introduction of the grid impedance, the sum of the gridconnected voltage and the grid impedance voltage is the capacitor voltage, so $\mathrm{Uz}^{*}$ is added to the control structure diagram. When the grid impedance is 0 , it is the traditional droop control strategy, and $\mathrm{Uz}^{*}$ can also be deleted. Meanwhile, in order to ensure the steady-state accuracy of grid-connected voltage, the controller of the voltage loop is designed as a proportional integral (PI) controller, where Kup is the proportional gain parameter of the voltage loop and Kui is the integral gain parameter of the voltage loop. In order to ensure the dynamic response speed of the system, the current loop controller is designed as a proportional $(\mathrm{P})$ controller, in which Kip is the proportional gain parameter of the current loop.

If Icf* is taken as the input, Icf as the output, and all other inputs are set to 0 , the transfer function of the current loop can be obtained, as shown in Equation (8):

$$
\mathrm{Ic}_{\mathrm{f}}=\frac{\frac{\mathrm{U}_{\mathrm{dc}}}{2} \cdot \mathrm{K}_{\mathrm{ip}} \mathrm{C}_{\mathrm{f}} \mathrm{s}}{\mathrm{L}_{\mathrm{f}} \mathrm{C}_{\mathrm{f}} \mathrm{s}^{2}+\frac{\mathrm{U}_{\mathrm{dc}}}{2} \cdot \mathrm{K}_{\mathrm{ip}} \mathrm{C}_{\mathrm{f}} \mathrm{s}+1} \mathrm{Ic}_{\mathrm{f}}^{*}-\frac{\mathrm{L}_{\mathrm{f}} \mathrm{C}_{\mathrm{f}} \mathrm{s}^{2}}{\mathrm{~L}_{\mathrm{f}} \mathrm{C}_{\mathrm{f}} \mathrm{s}^{2}+\frac{\mathrm{U}_{\mathrm{dc}}}{2} \cdot \mathrm{K}_{\mathrm{ip}} \mathrm{C}_{\mathrm{f}} \mathrm{s}+1} \mathrm{I}_{0}
$$

As the larger the frequency band bandwidth of the transfer function, the faster the response speed of the system, so the current loop should have as large a bandwidth as possible, so as to make the response speed faster. In addition, the amplitude-frequency characteristics of Icf/Io are required to be as close to 0 as possible within the bandwidth to reduce the influence of the current of the filter capacitance on the system. Next, the transfer function of the equivalent output impedance of the gridconnected pv inverter is established. Since the equivalent output impedance can be obtained by $\mathrm{Uo} / \mathrm{Io}$, both $\mathrm{Uo}^{*}$ and $\mathrm{Uz}^{*}$ in Figure 3 are set to 0 , thus the transfer function of the equivalent output impedance of the grid-connected PV inverter is obtained, as shown in Equation (9).

$$
G_{Z}(s)=\frac{C_{f} L_{f} L_{g} s^{4}+C_{f} L_{f} R_{g} s^{3}+L_{f} s^{2}}{C_{f} L_{f} s^{3}+\frac{U_{\mathrm{dc}}}{2} \cdot K_{i p} \cdot C_{f} s^{2}+S+\frac{U_{\mathrm{dc}}}{2} \cdot K_{i p} \cdot K_{u p} \cdot s+\frac{U_{\mathrm{dc}}}{2} K_{u p} \cdot K_{u i}}
$$


It can be seen from Equation (9) that, after the introduction of grid impedance, the equivalent output impedance of the grid-connected inverter using drooping control method is simultaneously subject to filter parameters. The equivalent output impedance is mainly affected by the network impedance and the PI controller parameters. Among them, the filter parameters are usually fixed, so it can be seen that the equivalent output impedance is mainly affected by the network impedance and PI controller parameters.

In order to analyze the influence of proportional parameters and integral parameters on the equivalent output impedance of grid-connected inverter in the frequency domain, the corresponding frequency characteristic curves are obtained under the two conditions of constant proportional parameters but varying integral parameters, and constant integral parameters but varying proportional parameters. As shown in Figure4 and Figure5, the Bode diagram when the integral parameters change and the Bode diagram when the proportion parameters change respectively.

The Bode diagrams in Figure 4 and 5, the amplitudefrequency characteristics of the upper part and the phasefrequency characteristics of the lower part respectively represent the trend of amplitude and impedance Angle of the grid-connected inverter equivalent output impedance changing with frequency. In this paper, the lower part of Bode is mainly concerned. The common feature of the two figures is that the impedance Angle starts from $180^{\circ}$, which presents resistance, and gradually decreases to around $90^{\circ}$, which presents sensibility, as the frequency increases. Then, near the angular frequency of $103 \mathrm{rad} / \mathrm{s}$, the network impedance begins to affect the impedance Angle with the increase of frequency, which causes the impedance Angle to rapidly change from the perceptual $90^{\circ}$ to the capacitive $270^{\circ}$. Finally, the impedance Angle will gradually change from the capacitive $270^{\circ}$ to the capacitive $90^{\circ}$ with the increasing frequency.

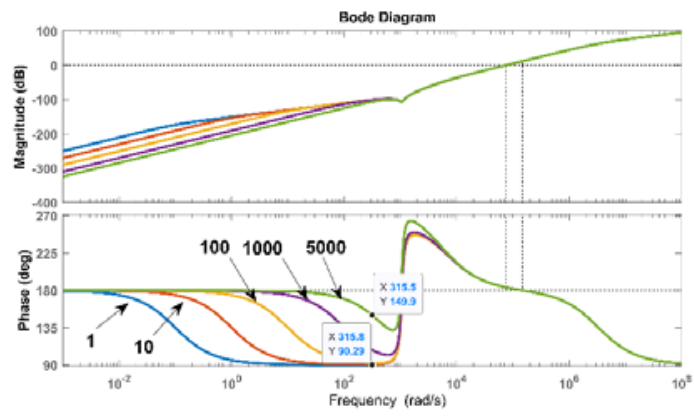

Figure 4. Bode diagram of integration parameter variation

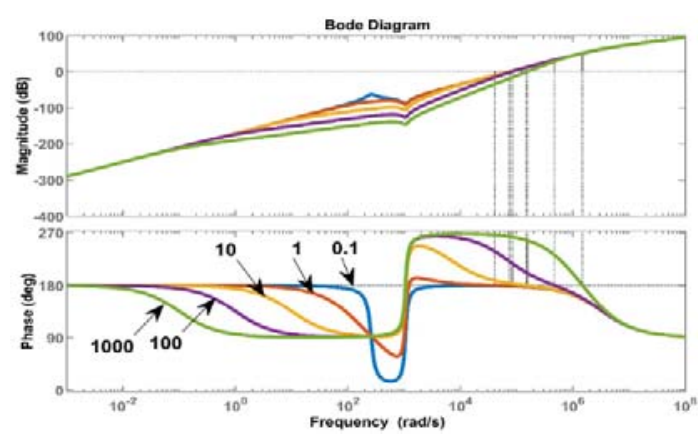

Figure 5. Bode diagram of gain parameter variation

As can be seen from Figure 4, when the proportion parameter remains unchanged, the band width area of perceptual $90^{\circ}$ becomes smaller and smaller with the continuous increase of integral parameter. When the integral parameter changes from 1 to 5000 , the impedance angle near the angular frequency of $314 \mathrm{rad} / \mathrm{s}$, namely the power grid frequency of $50 \mathrm{HZ}$, also changes from about 90 perceptively to about 150 resistively, which gradually fails to meet the prerequisite requirements of P-f and Q-U droop characteristics. As can be seen from Figure 5, when the integral parameter remains unchanged, the band width area of perceptual $90^{\circ}$ becomes larger and larger with the increasing proportion parameter.

Appropriate proportion and integral parameters can be obtained by taking into account the influence of the above proportion parameters and integral parameters on the phase-frequency characteristics of the equivalent impedance and the size of the perceptual bandwidth area of $90^{\circ}$. In this paper, within the range of $100 \mathrm{rad} / \mathrm{s}$ to $500 \mathrm{rad} / \mathrm{s}$, the expected impedance Angle can be limited to $88^{\circ}$ to $92^{\circ}$, so that the equivalent output impedance can be strictly limited to the vicinity of the perceptual state, and the bandwidth is large. Specifically, the proportional parameter Kup and the integral parameter Kui that meet the requirements can be obtained by solving the equations shown in equation (10) below.

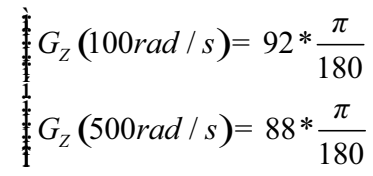

\section{Hardware in - loop real-time simulation and result analysis}

In order to further verify the correctness of the improved droop control algorithm based on impedance detection proposed in this paper, the hardware test system RT-LAB is adopted to verify the algorithm. Figure 6 is the schematic diagram of the test platform. Firstly, the photovoltaic grid-connected power generation system mentioned above is realized in RT-LAB through PC upper computer. Then the proposed improved drooping control algorithm based on power grid impedance detection is implemented in TI's digital processor TMS320F28335DSP.

At last, the communication between RT-LAB and DSP can be realized by using the $\mathrm{I} / \mathrm{O}$ board card and many $\mathrm{I} / \mathrm{O}$ 
channels and AD/DA conversion channels. The oscilloscope can detect the output power, voltage and current of grid-connected inverter in real time. PC upper computer is used to realize the control of the whole platform.

Set in the validation test, the test time $\mathrm{t}=2 \mathrm{~s}$. During $\mathrm{t}$ $=0 \sim 0.8 \mathrm{~s}$, the initial grid impedance is $\mathrm{Rg}=0.3 \Omega$, $\mathrm{Lg}=0.6 \times 10-3 \mathrm{H}$, the value $\mathrm{Rg} / \mathrm{XLg}$ equal 1.5915 , presenting perceptual. When $\mathrm{t}=0.8 \mathrm{~s}$, grid impedance mutation for $\mathrm{Rg}=0.6 \Omega, \mathrm{Lg}=1 \times 10-3 \mathrm{H}$, the $\mathrm{Rg} / \mathrm{XLg}$ value of 19.0986 , presents the resistance.

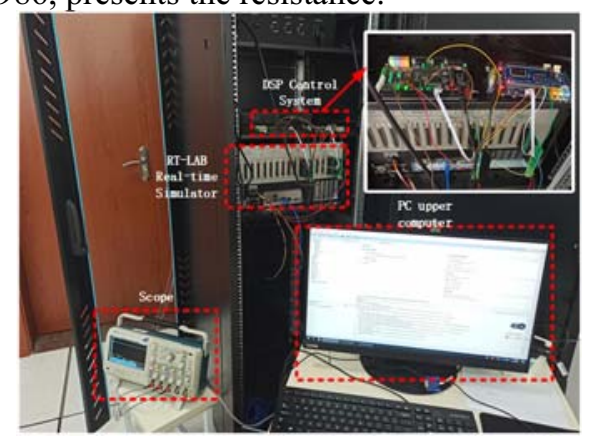

Figure 6. Test platform diagram

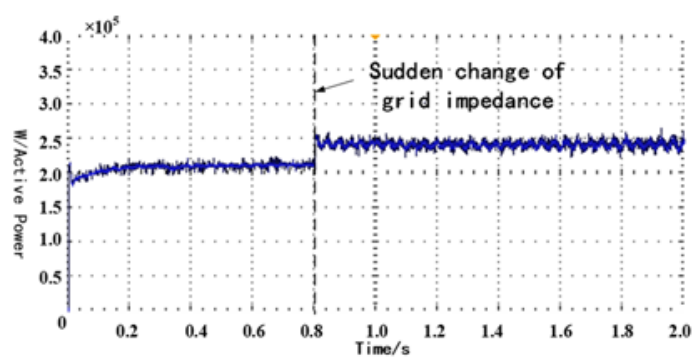

Figure 7. Grid-connected power without improved droop control

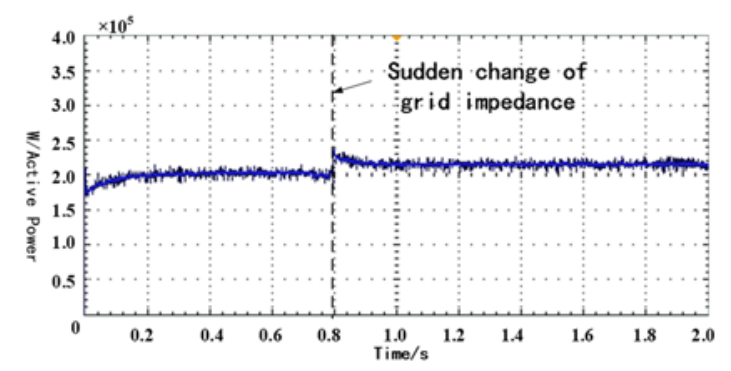

Figure 8. Grid-connected power with improved droop control

Among them, when the improved droop control algorithm is not adopted, the grid-connected power of the photovoltaic grid-connected inverter is shown in Figure 7.

It can be seen from Figure 13 that during $\mathrm{t}=0 \sim 0.8 \mathrm{~s}$, the grid-connected inverter can steadily output about $200 \mathrm{KW}$ rated grid-connected power. When $\mathrm{t}=0.8 \mathrm{~s}$, as the inductive impedance of the power grid changes to resistive impedance, the grid-connected output power of the gridconnected inverter increases by about $250 \mathrm{KW}$, and its increment is about $50 \mathrm{KW}$, accounting for $25 \%$ of the rated power and causing serious extra loss of active power. In addition, the output grid-connected power cannot be maintained constant, but there is a situation of reciprocating oscillation, whose amplitude can be seen to be about $230 \mathrm{KW}$ to $260 \mathrm{KW}$. When the $\mathrm{Rg} / \mathrm{Xg}$ value of the power grid impedance is larger, that is, the more inductive the power grid impedance is, the larger the oscillation amplitude will be. For this reason, the improved droop control algorithm proposed in this paper should be adopted, and the grid-connected power diagram using the improved droop control is shown in Figure 8.

As can be seen from the Figure 8 , after $\mathrm{t}=0.8 \mathrm{~s}$, the output of the photovoltaic grid inverter grid power is no longer a significant reciprocating oscillation appears, at about $215 \mathrm{kw}$, the increment of about $15 \mathrm{kw}$, about 7.5\%, than the rated power than with the improved droop control percentage increase in the size of than dropped by about $17.5 \%$ and its power in power grid impedance increase after mutation from about $50 \mathrm{kw}$ in Figure 13 to about 15 $\mathrm{kw}$, fell more than $70 \%$, significantly reduced the loss of active power. This result indicates that the improved drooping control algorithm proposed in this paper has successfully suppressed the reciprocating oscillation and other unstable situations when the power network impedance mutates to resistance, and improved the power quality and system stability of photovoltaic gridconnected power generation system.

\section{Conclusion}

In this paper, an improved droop control algorithm based on grid impedance is proposed in view of the influence of abrupt power grid impedance on the output power stability of photovoltaic grid-connected power generation system using droop control. By introducing the power network impedance into the traditional droop control, a new double-loop control structure diagram of voltage and current is designed, and the influence of power network impedance on droop control is analyzed theoretically. Then, using DFT method to obtain the grid impedance, adaptively updated in real time the control parameters of PI controller, realized the improved droop control algorithm based on impedance detection. In off-line simulation and hardware in loop real-time simulation, the improved control strategy is applied in photovoltaic grid power generation system, the results show that the method can be in the power grid impedance mutation, adaptive change the control parameters of droop control, the realization of the aim of photovoltaic grid inverter can output stable.

\section{References}

1. Zhu Huimin, Yuan Shun, Li ChunLai. A Reactive voltage control strategy for Photovoltaic power station based on variable sag parameters [J]. Renewable Energy,202,38(08):1103-1108.

2. Liu Cheng, Wang Hui, LI TianHui, Jin Xue, Li Zhe. Research on the influence of distributed new energy generation on the voltage of distribution network [J]. Renewable energy,2019,37(10):1465-1471.1

3. T. Roinila and T. Messo, "Online Grid-Impedance Measurement Using Ternary-Sequence Injection," in IEEE Transactions on Industry Applications, vol. 54, 
no. 5, pp. 5097-5103, Sept.-Oct. 2018.

4. Yang Ming, Zhou Lin, ZHANG DongXia, Zhang Mi. Analysis of grid-connected stability of large photovoltaic power stations considering the influence of grid impedance $[\mathrm{J}]$. Journal of electrotechnical technology,2013,28(09):214-223.

5. Zhou Lin, Zhang Mi, Ju XiuLi, He GuoQing. Analysis of the influence of grid impedance on the stability of large-scale grid-connected pv system [J]. Chinese journal of electrical engineering, 2013,33(34) :34-41+9. 